Revista Brasileira de Agricultura Irrigada v.7, n.2, p.91-101, 2013

ISSN 1982-7679 (On-line)

Fortaleza, CE, INOVAGRI - http://www.inovagri.org.br

DOI: $10.7127 /$ rbai.v7n200105

Protocolo 105.12 - 06/11/2012 Aprovado em 10/04/2012

\title{
EVOLUÇÃO DA QUALIDADE DA IRRIGAÇÃO APÓS AS ATUAÇÕES DO SERVIÇO DE ASSESSORAMENTO AO IRRIGANTE
}

\author{
Sílvio Carlos Ribeiro Vieira Lima ${ }^{\mathbf{1}}$; José Antônio Frizzone ${ }^{2}$; \\ Aristides Martins dos Santos Neto ${ }^{3}$; José Aguiar Beltrão Júnior ${ }^{4}$; \\ Francisco Mourão Rodrigues Júnior ${ }^{5}$; José Lúcio Nascimento Nunes Filho ${ }^{6}$
}

\section{RESUMO}

O Serviço de Assessoramento ao Irrigante - SAI é um projeto pioneiro que está sendo executado no Distrito de Irrigação Baixo Acaraú - DIBAU, Ceará. O principal objetivo é transferir a informação ao irrigante através de mensagem de celular, e-mail e pela própria página na internet. O SAI possui uma equipe de técnicos que monitora constantemente a qualidade da irrigação referente ao manejo do tempo de irrigação e do uso adequado do sistema de irrigação. Para se irrigar bem é preciso ter o sistema funcionando adequadamente, pois desta forma haverá uma boa qualidade da irrigação. Alguns lotes vêm sendo monitorado desde a criação do SAI e este trabalho objetivou avaliar a qualidade da irrigação destes irrigantes. Concluiu-se que ocorre um aumento da qualidade quando se inicia a atuação do SAI com o irrigante e esta atuação é primordial para a melhoria da uniformidade de emissão dos sistemas $\mathrm{e}$ consequentemente da eficiência da irrigação. O SAI deve ser utilizado como um importante instrumento de manejo da irrigação em áreas irrigadas.

Palavras-chave: Manejo da Irrigação; Software; Eficiência da Irrigação, Uniformidade;

\section{EVOLUTION OF THE IRRIGATION QUALITY AFTER THE ACTUATIONS OF THE ADVISORY SERVICE FOR IRRIGATOR}

\footnotetext{
ABSTRACT

${ }^{1}$ Pesquisador do Instituto INOVAGRI, silviocarlos@inovagri.org.br.

${ }^{2}$ Professor Titular, ESALQ/USP

${ }^{3}$ Tecnólogo em Irrigação, Pesquisador do INOVAGRI

${ }^{4}$ Engenheiro Agrônomo, Pesquisador do INOVAGRI

${ }^{5}$ Tecnólogo em Irrigação, Pesquisador do INOVAGRI

${ }^{6}$ Engenheiro Agrônomo, Pesquisador do INOVAGRI
}

The Advisory Service for irrigator - SAI is a pioneering project that is running in Baixo Acaraú Irrigation District - DIBAU Ceará. The main goal is to transfer the information to the irrigator through mobile message, e-mail and the web page itself. The SAI has a 


\section{EVOLUÇÃO DA QUALIDADE DA IRRIGAÇÃO APÓS AS ATUAÇÕES DO SERVIÇO DE ASSESSORAMENTO AO IRRIGANTE}

team of technicians that constantly monitors the quality of irrigation for the management of irrigation time and the proper use of the irrigation system. To irrigate good, is necessary to have the system working properly, because this way there is good irrigation quality. Some lots have been monitored since the creation of SAI and this study aimed to evaluate the quality of irrigation these lots. It was concluded that there is an increase in quality when it begins the SAI's work with irrigator and this activity is essential for improving emission uniformity of systems and consequently the irrigation efficiency. The SAI should be used as an important tool for irrigation management in irrigated areas.

Keywords: Irrigation Management; Software; Irrigation Efficiency, Uniformity

\section{INTRODUÇÃO}

Dentre os perímetros irrigados, localizados no Ceará, o Distrito de Irrigação do Baixo Acaraú - DIBAU é o segundo maior, em termos de demanda hídrica. Apesar da elevada demanda de água para irrigação, o DIBAU não possuía nenhuma estratégia de manejo da mesma até a implantação do Projeto Serviço de Assessoramento ao Irrigante - SAI (SANTOS NETO et al., 2011; LIRA et al., 2011 e LIMA et al., 2012). Tal fato levava os irrigantes a manejar a irrigação pelo total empirismo de outras experiências, sem levar em consideração as peculiaridades locais.

O SAI tem como objetivo dar respostas às demandas tecnológicas dos irrigantes, ou seja, realizar um assessoramento sobre o manejo da irrigação. De acordo com (LIMA et al. 2012), as primeiras experiências com serviços de assessoramento ao irrigante foram realizadas nos Estados Unidos. O CIMIS (California Irrigation Management Information System), provavelmente é quem melhor representa o potencial dos SAIs. Outros autores estudaram SAIs e demonstram a importância deles para uma irrigação eficiente (LORITE et al. 2012; LIMA et. al. 2010; CÓRCOLES et al. 2010; GONZÁLEZ-DUGO et al. 2008; ORTEGA et al. 2005; MONTORO et al. 2011; CAR et al. 2012).
O DIBAU possui vocação para a fruticultura e seu projeto foi concebido para utilizar a microirrigação (gotejamento e microaspersão). Este Distrito, de acordo com Lira et al. (2011), possui 95\% de sua área ocupada com este método.

A microirrigação, por ser um método de elevada eficiência de uso da água é particularmente recomendado para áreas com escassez desse recurso ou quando a cobrança pelo uso deste onera o processo produtivo. Quando adequadamente projetados, instalados, mantidos e manejados, os sistemas de microirrigação podem eliminar os problemas advindos do escoamento superficial e a consequente erosão do solo e atingir elevados níveis de eficiência de aplicação e uniformidade de distribuição de água e produtos químicos. Logo, é unânime a concordância de que uma das maiores vantagens é a economia de água (FRIZZONE et al., 2012).

Mesmo com toda a tecnologia empregada, sistemas de microirrigação mal dimensionados, instalados inadequadamente ou manejados de forma errada podem implicar em baixos índices de uniformidade e eficiência, não justificando o alto valor investido neste equipamento.

Um serviço de apoio técnico ao irrigante pode contribuir, com a 


\section{EVOLUÇÃO DA QUALIDADE DA IRRIGAÇÃO APÓS AS ATUAÇÕES DO SERVIÇO DE ASSESSORAMENTO AO IRRIGANTE}

melhoria e até com o desaparecimento destes problemas em campo, mas, conforme Mateos (2008) existem várias limitações para este tipo de atividade e, alguns deles aparecem no início do serviço e outros surgem no desenvolvimento. Isto pode vir a limitar a sua eficácia.

A determinação da uniformidade de emissão de água dos sistemas de Irrigação (UE) aplicando a metodologia de Keller e Karmelli (1974) no DIBAU, foi iniciada por Santos Neto et al., (2011) que encontrou em alguns lotes baixa UE. Este autor demonstrou que somente com algumas recomendações e sugestões apresentadas pelos técnicos do SAI, todos os sistemas melhoraram a sua UE. Ao longo do Projeto SAI esta avaliação vem sendo monitorada com os mesmo irrigantes e nos mesmos locais.

Segundo Frizzone et al., (2012), tem-se expressado a qualidade da irrigação por alguns indicadores de desempenho nomeados, basicamente, por três palavras-chave: uniformidade, eficiência e grau de adequação. O termo uniformidade refere-se aos indicadores associados à variabilidade da lâmina de irrigação aplicada. É uma grandeza que

\section{MATERIAL E MÉTODOS}

O trabalho foi realizado no DIBAU, com sede localizada no Triangulo do Marco a $220 \mathrm{~km} \mathrm{de}$ Fortaleza, na região Norte do estado do Ceará. O clima da região está caracterizado por Santos Neto et al., (2011) e Lima et. al., (2012). Os dados para determinar a ETo diariamente foram obtidos da estação do Instituto Nacional de Meteorologia que está instalada no Distrito. A determinação da ETo foi feita utilizando-se a equação de caracteriza todo o sistema de irrigação e intervém no seu projeto, tanto agronômico, pois afeta o cálculo da quantidade de água necessária à irrigação, quanto no hidráulico, pois, em função dela, define-se o espaçamento entre emissores de água, a vazão do sistema e o tempo de irrigação.

O irrigante para realizar uma boa aplicação de água às plantas precisa ter conhecimento das seguintes informações: quando (tempo), quanto (lâmina) e como (método-sistema) irrigar. Se ele possui os dois primeiros, mas o sistema não está sendo bem utilizado, podemos considerar que ele tem uma baixa qualidade da irrigação. Certamente, qualquer um dos três fatores interferirá na qualidade, mas neste trabalho, focaremos somente no sistema. Se o irrigante melhora a utilização do sistema de irrigação, ele terá uma melhoria da qualidade da irrigação daquele Distrito.

O objetivo desta pesquisa foi verificar a evolução da qualidade da irrigação, através das avaliações realizadas pela equipe do Serviço de Assessoramento ao Irrigante - SAI e de sua atuação no apoio ao irrigante do DIBAU.

Penman-Monteith (ALLEN et al., 1998).

Para a realização do manejo da irrigação do SAI, em 2011 foi desenvolvido um software específico para o SAI. O sistema de computação criado é o Sistema de Assessoramento ao Irrigante - s@i. O s@i é um sistema Web e o fluxograma do processo de envio do SMS está apresentado na Figura 1. A divisão de cultivos do DIBAU gerada pelo s@i pode ser visualizada na Figura 1. 


\section{EVOLUÇÃO DA QUALIDADE DA IRRIGAÇÃO APÓS AS ATUAÇÕES DO SERVIÇO DE ASSESSORAMENTO AO IRRIGANTE}

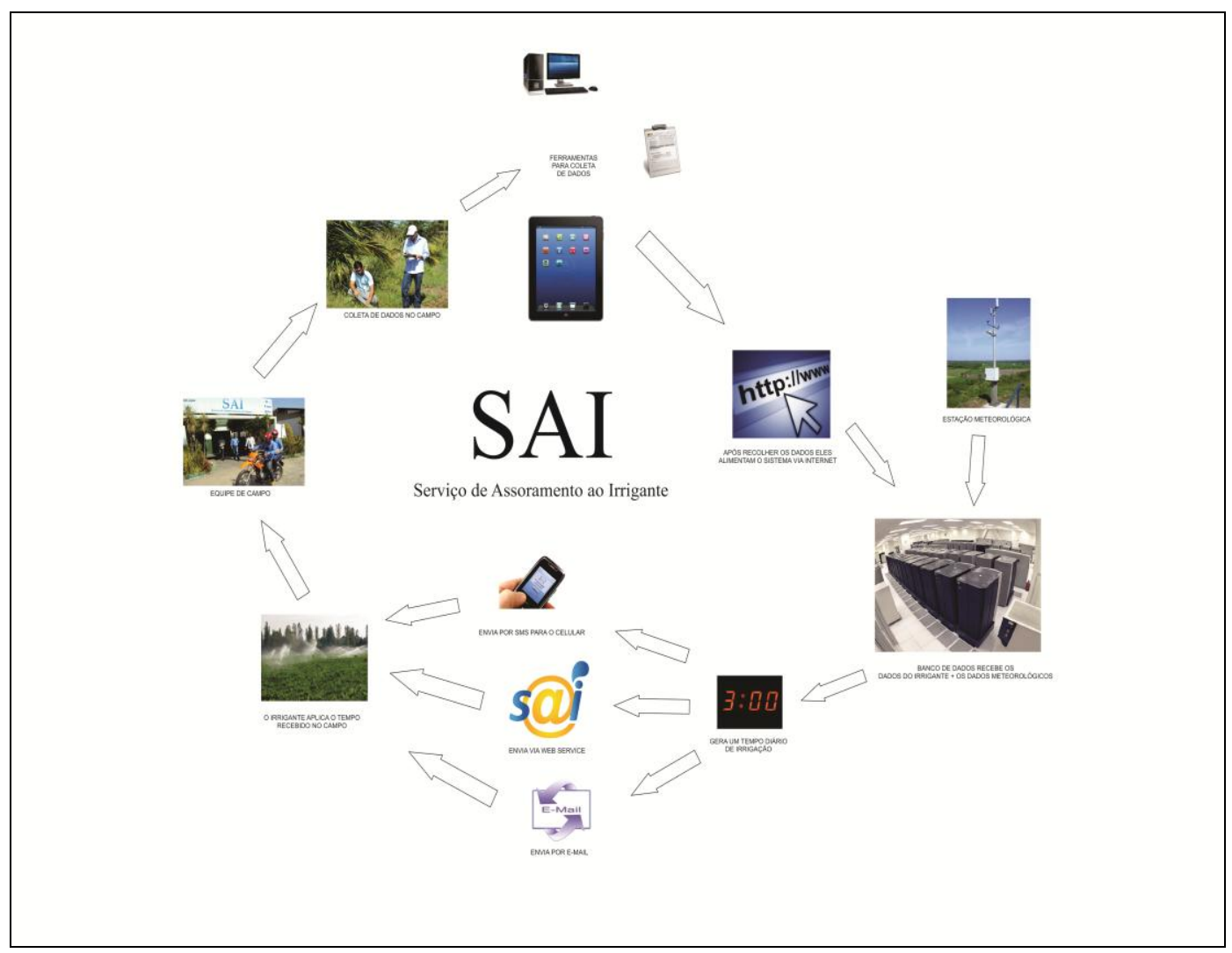

Figura 01. Fluxograma do processo do Sistema s@i

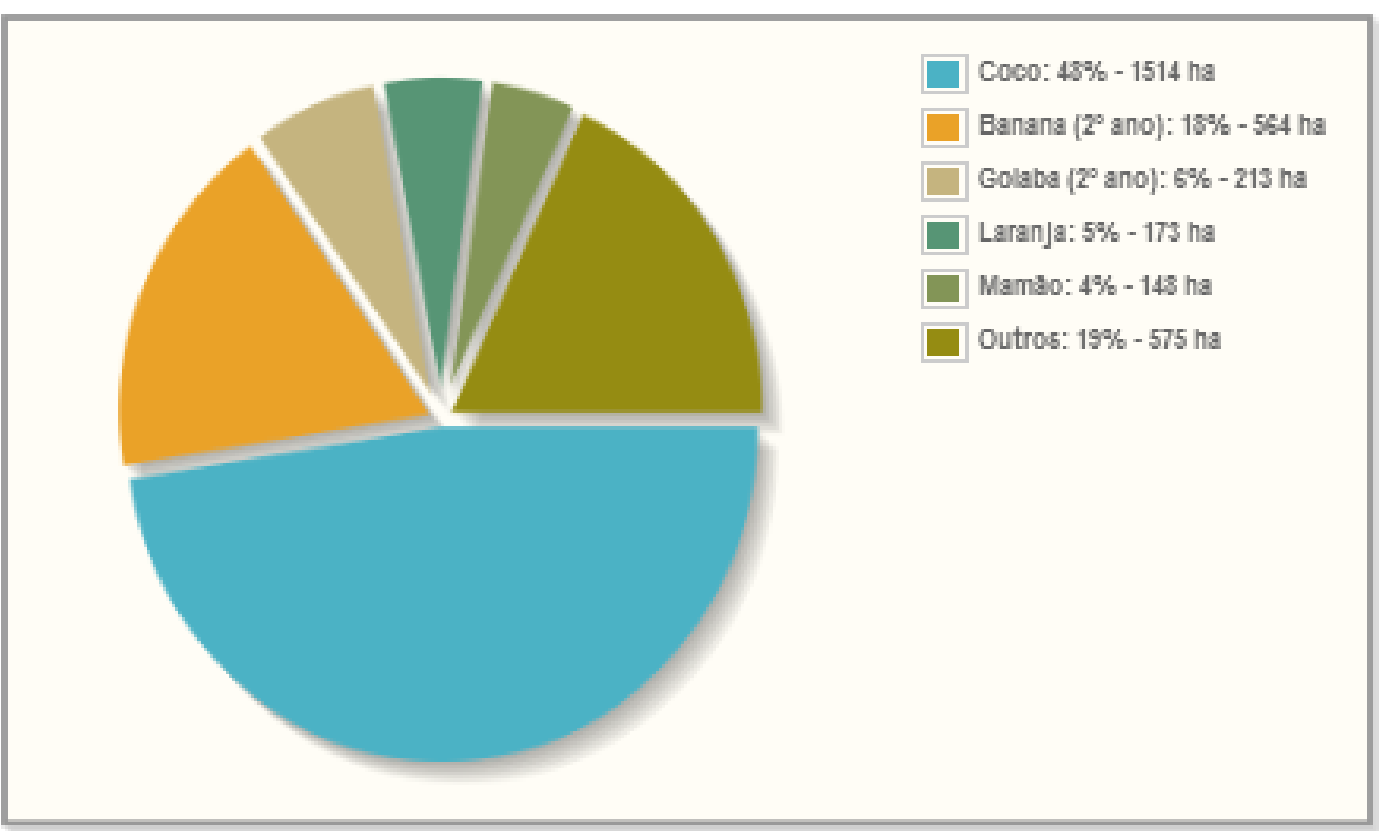

Figura 2. Divisão de cultivos no DIBAU conforme o Sistema s@i

O software funciona na web, sem a necessidade de instalação em computador. Isto facilita a utilização dele por agricultores em lugares 


\section{EVOLUÇÃO DA QUALIDADE DA IRRIGAÇÃO APÓS AS ATUAÇÕES DO SERVIÇO DE ASSESSORAMENTO AO IRRIGANTE}

diversos do país ou do mundo, bastando apenas ter o login e senha.

O Sistema $\mathrm{s} @ \mathrm{i}$ possui um módulo de irrigação que utiliza a base de dados do módulo de assessoramento integrado a uma estação meteorológica automática para calcular e enviar, por meio de mensagens de SMS ou E-mail, o tempo de irrigação, diário de cada plantio. Este módulo também calcula a uniformidade de emissão do sistema com os dados coletados pelo técnico, ou até mesmo pelo irrigante que alimenta o sistema.

De acordo com Frizzone (2007), a eficiência de aplicação de água na parcela (EA) é definida pela relação entre usos benéficos de água na parcela e quantidade total de água aplicada. Em uma irrigação plena (sem déficit), a IRN será a lâmina mínima de irrigação infiltrada na área para satisfazer a evapotranspiração no período. Se considerarmos que um sistema de microirrigação não aplicará a mesma quantidade de água em todos os pontos da área, no cálculo da irrigação total necessária, deve ser considerada a UE. $\mathrm{Na}$ irrigação sem déficit a lâmina mínima de água infiltrada na área deve ser IRN e, neste caso, a uniformidade de emissão pode ser definida pela relação entre IRN e a lâmina média infiltrada. Para que a fração da área menos irrigada receba IRN a lâmina média aplicada deverá considerar a UE, sendo ITN calculada da seguinte forma:

$$
\mathrm{ITN}=\frac{\mathrm{IRN}}{\mathrm{EA} \times \mathrm{UE}}
$$

para o seguinte caso: sendo as perdas de água por percolação maiores que a fração de lixiviação considera-se que não há necessidade de lâmina adicional para controle da salinidade e que as perdas por percolação são suficientes para promover a lavagem da zona radicular e manter o nível de salinidade em condição não prejudicial. Também, deve-se considerar que a EA será igual a 1 o que deverá ser considerado, para temos práticos de gestão de um número grande de irrigantes, apenas a UE. Então, a irrigação total necessária (ITN) será definida da seguinte forma:

$$
\mathrm{ITN}=\frac{\mathrm{IRN}}{\mathrm{UE}}
$$

As avaliações foram realizadas em 07 períodos diferentes de setembro de 2011 a novembro de 2012 com os mesmo 30 irrigantes, dos 44 avaliados por Santos Neto et al., (2011). Estes 30 estão sendo acompanhados permanentemente desde 2011.

A equipe foi formada por três técnicos em cada avaliação. Um foi responsável pela marcação do sistema, ou seja, onde seriam os pontos de coletas de pressão e vazão, outro responsável pela coleta e outro pela marcação do tempo e anotação. As anotações eram feitas com lápis, em uma planilha e posteriormente inseridas nos@i.

Na Figura 3 pode ser visualizada a tela do s@i. Na Figura 3a está apresentada a Tela Fazenda, que faz a divisão da propriedade em setores com os cultivos. O item abaixo da figura da cultura (Ver setor 1) representa a eficiência com a metodologia de cálculo da UE por Keller e Karmelli (1974). Na Figura $3 b$ está apresentado o passo seguinte que é a planilha de cálculo onde podem ser inseridos os dados e o software gera o resultado e já adiciona ao cálculo do ITN daquele setor.

Após a conclusão das análises foi feita uma nova avaliação dos lotes e uma atualização cadastral. 
EVOLUÇÃO DA QUALIDADE DA IRRIGAÇÃO APÓS AS ATUAÇÕES DO

SERVIÇO DE ASSESSORAMENTO AO IRRIGANTE

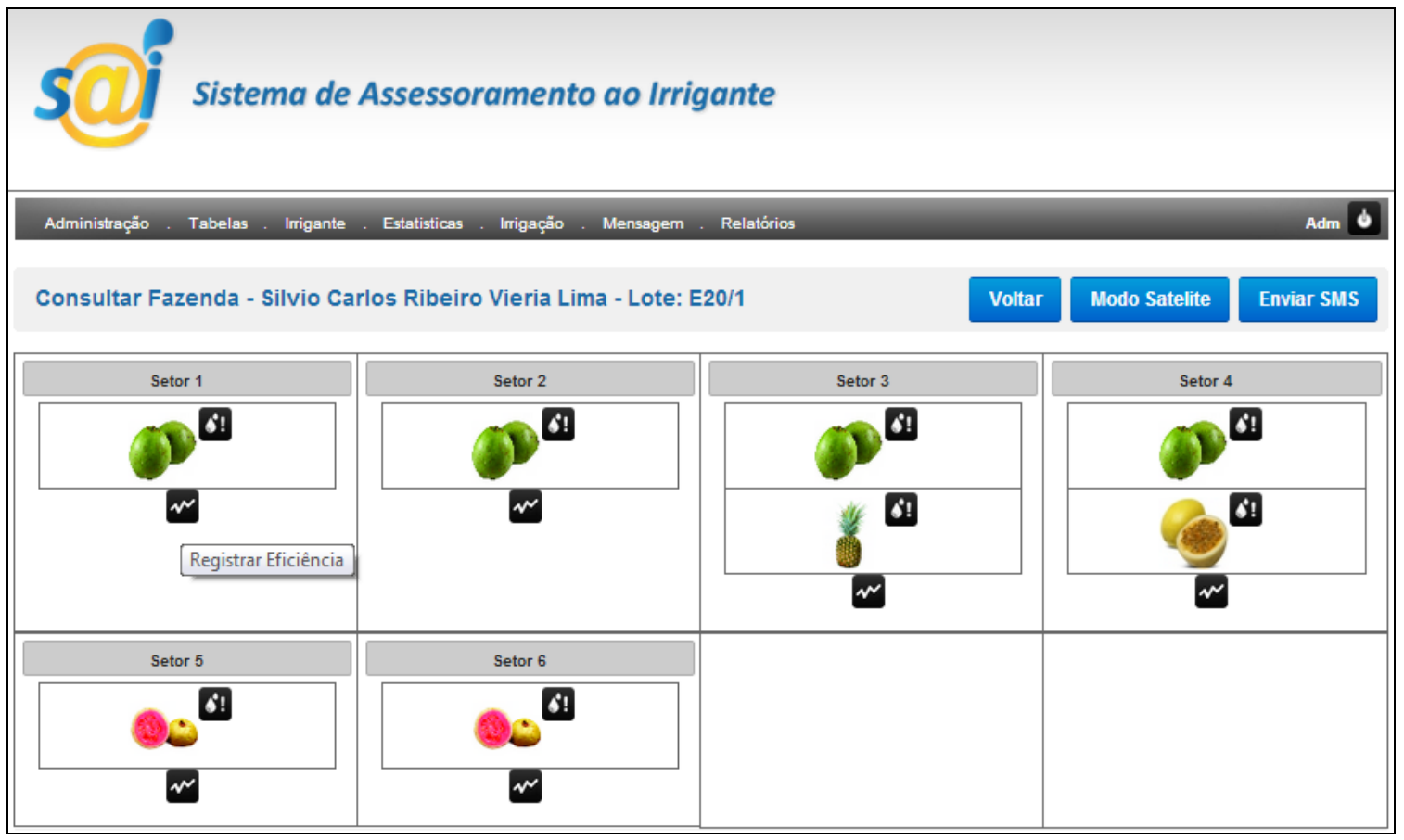

Figura 03.a. Tela Fazenda do s@i com a representação dos cultivos

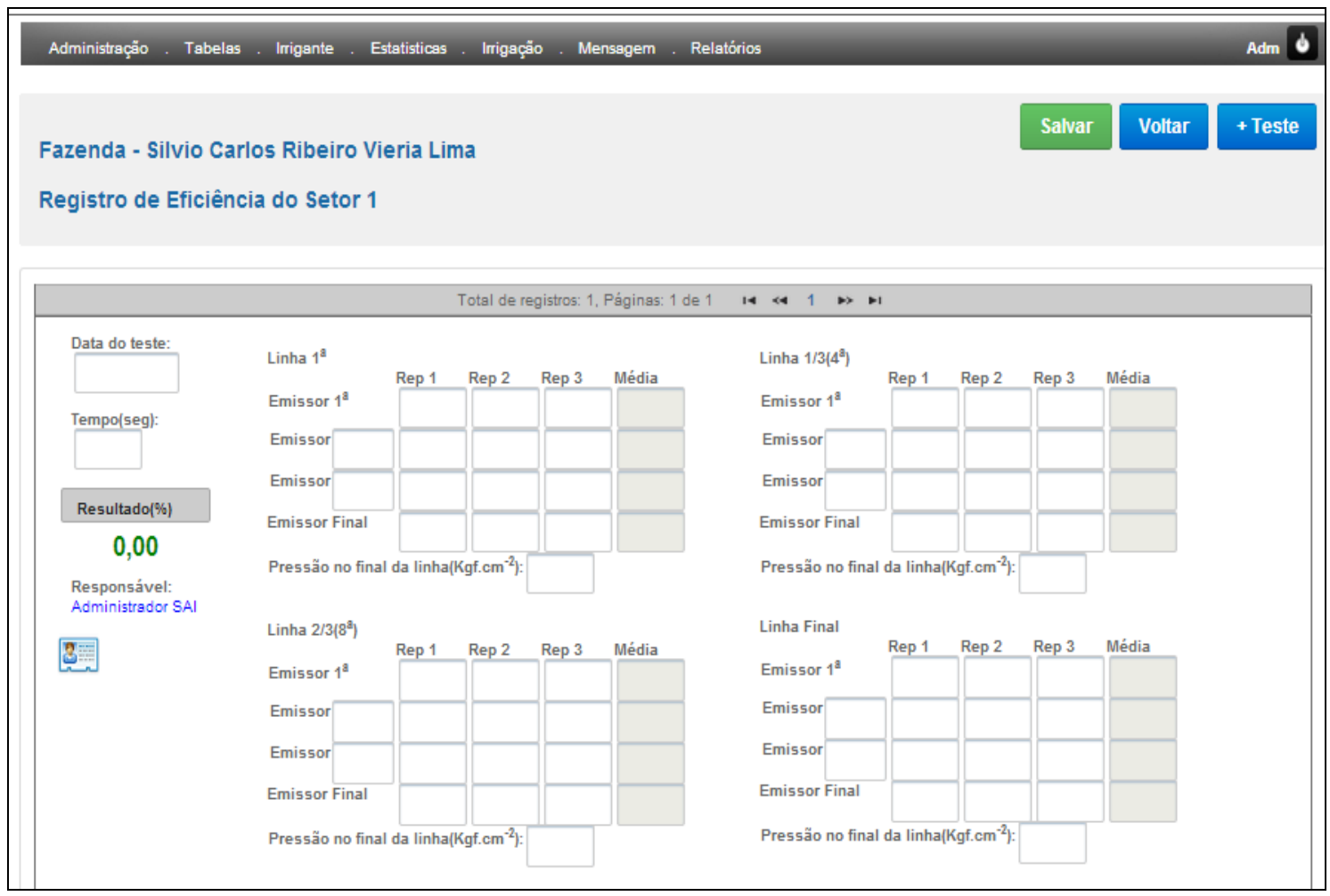

Figura 03.b. Tela Eficiência do s@i para o cálculo da UE por (Keller e Karmelli, 1974) 


\section{EVOLUÇÃO DA QUALIDADE DA IRRIGAÇÃO APÓS AS ATUAÇÕES DO SERVIÇO DE ASSESSORAMENTO AO IRRIGANTE}

\section{RESULTADOS E DISCUSSÃO}

Em relação às avaliações dos sistemas, os resultados estão apresentados na Tabela 1 conforme a classificação de Bralts (1986). Observase que $15,49 \%$ dos irrigantes avaliados possuem uma excelente Uniformidade de emissão, porém a maior parte, $49,30 \%$ ficou concentrada na classificação de "bom". Isto se justifica pela condição de que todos os lotes foram avaliados com microaspersão, $o$ que seria um valor esperado e aceitável.
Nota-se uma evolução em relação ao encontrado por Santos Neto et al., (2011), pois no estudo daquele autor, existia uma dispersão entre todas as classificações, com "ruim" ficando com $20 \%$ (com valores chegando ao mínimo de $9,20 \%)$ e "excelente" em $27 \%$ dos valores, pois existiam sistemas de gotejamento, o que não foi o caso deste estudo. Nesta pesquisa, na classificação de regular tivemos $15,49 \%$ e ruim $19,72 \%$.

Tabela 1. Porcentagem de lotes avaliados conforme a classificação de Bralts (1986).

\begin{tabular}{ccc}
\hline $\mathrm{N}^{\mathbf{0}}$ de Avaliações & Classificação de U.E & \% dos lotes \\
\hline 11 & Excelente & 15,49 \\
35 & Bom & 49,30 \\
11 & Regular & 16,90 \\
14 & Ruim & 18,31 \\
\hline 71 & & 100,00 \\
\hline
\end{tabular}

Na Figura 4 está apresentada a UE por cultura e a relação pelo número de microaspersores por ha. A melhor UE foi obtida pela cultura da graviola seguida pelo Coco. Quando se consideram vários emissores formando uma unidade, como é o caso de emissores agrupados para irrigar uma única árvore, a uniformidade de aplicação de água é bastante melhorada, comparada à utilização de um único emissor por árvore (WU, 1997) e (WU et al., 2007) citado por Frizzone et al., (2012). Este autor comenta que o entupimento dos emissores, seguido pelo espaçamento entre eles, são os fatores mais significativos que afetam a uniformidade espacial da irrigação, mas o agrupamento de emissores é tão significativo quanto o espaçamento.

Quando observamos a cultura da banana com um microaspersor para quatro plantas, identificamos uma menor UE. Certamente a EA será bem menor neste caso específico, pois ocorrerão diversas perdas por percolação, evaporação e deriva pelo vento.

As demais culturas goiaba e acerola tiveram os menores resultados de EU. Na acerola é mais grave, pois possui um alto valor de microaspersores por hectare, o que também irá refletir na eficiência de aplicação. 


\section{EVOLUÇÃO DA QUALIDADE DA IRRIGAÇÃO APÓS AS ATUAÇÕES DO SERVIÇO DE ASSESSORAMENTO AO IRRIGANTE}

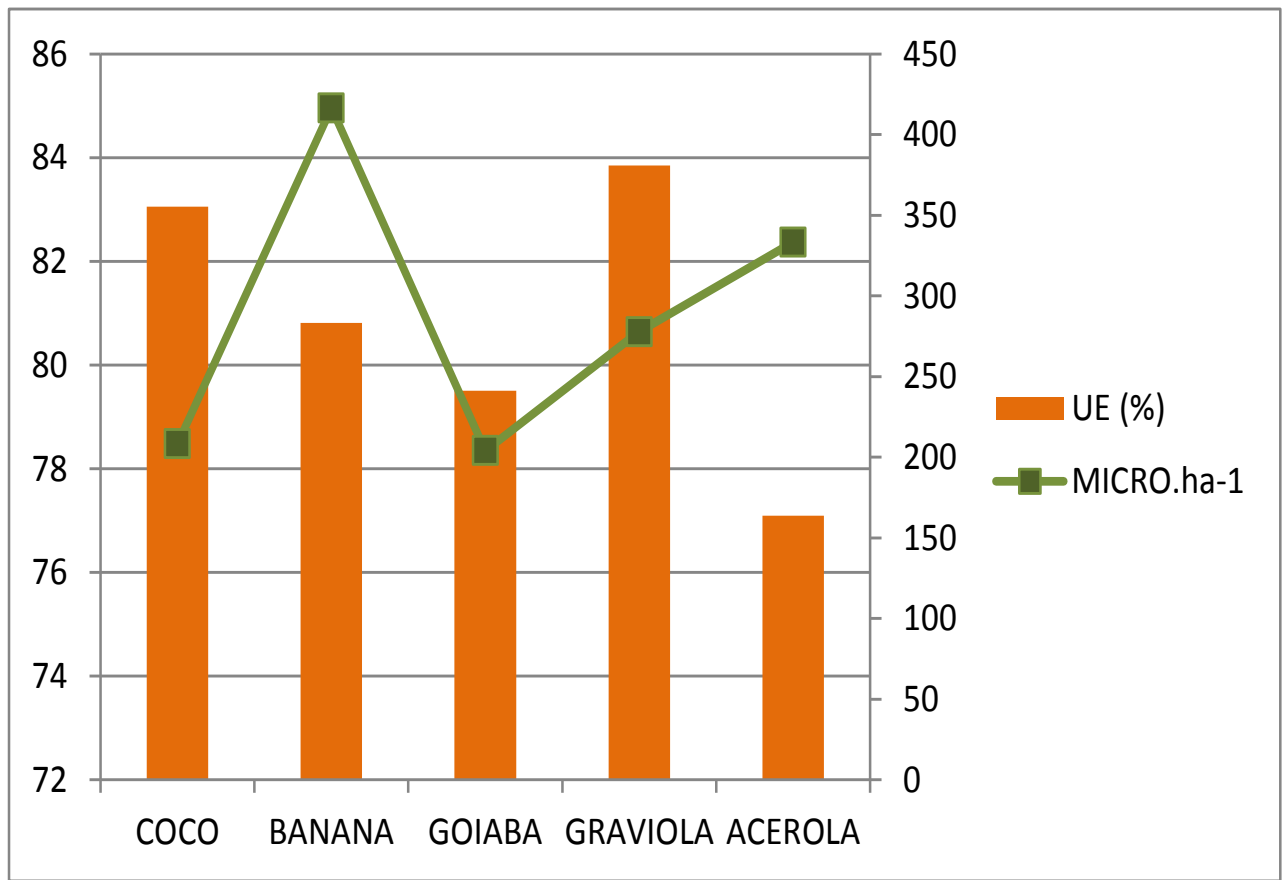

Figura 5. Relação cultura uniformidade e densidade de micro por hectare

Em relação ao período de avaliação, os resultados apresentados na Figura 5 mostram que uma baixa uniformidade é obtida no início da campanha da irrigação como pode ser observada nos meses de setembro de 2011 e agosto de 2012. Isto pode ser explicado, visto que todo o sistema ficou sem operação, pelo menos, nos últimos quatro meses, o que acarreta em falta de manutenção e limpeza. Frizzone et al. 2012 cita que um exemplo clássico é a escassa atenção dada à perda produtiva potencial por falhas provenientes da falta de manutenção dos sistemas de irrigação. As perdas de produtividade potencial nos projetos de irrigação, decorrentes da manutenção inadequada, são pouco discutidas na literatura.
Este período parado incorre também em um não assessoramento na operação do sistema, o que implicará, inicialmente, em um baixo rendimento do operacional. $\mathrm{O}$ operador ainda não está com habilidade suficiente para manejar corretamente o sistema de irrigação. Após o período inicial de operação e de assessoramento, a média da UE se eleva, como pode ser observado nos meses de Janeiro e setembro de 2012 da Figura 5.

Desta forma, podemos caracterizar como um aumento da qualidade da irrigação no DIBAU com a atuação do SAI. Esta importância também é relatada por (LORITE et al., 2012; LIMA et. al., 2010 e CAR et al., 2012). 


\section{EVOLUÇÃO DA QUALIDADE DA IRRIGAÇÃO APÓS AS ATUAÇÕES DO} SERVIÇO DE ASSESSORAMENTO AO IRRIGANTE

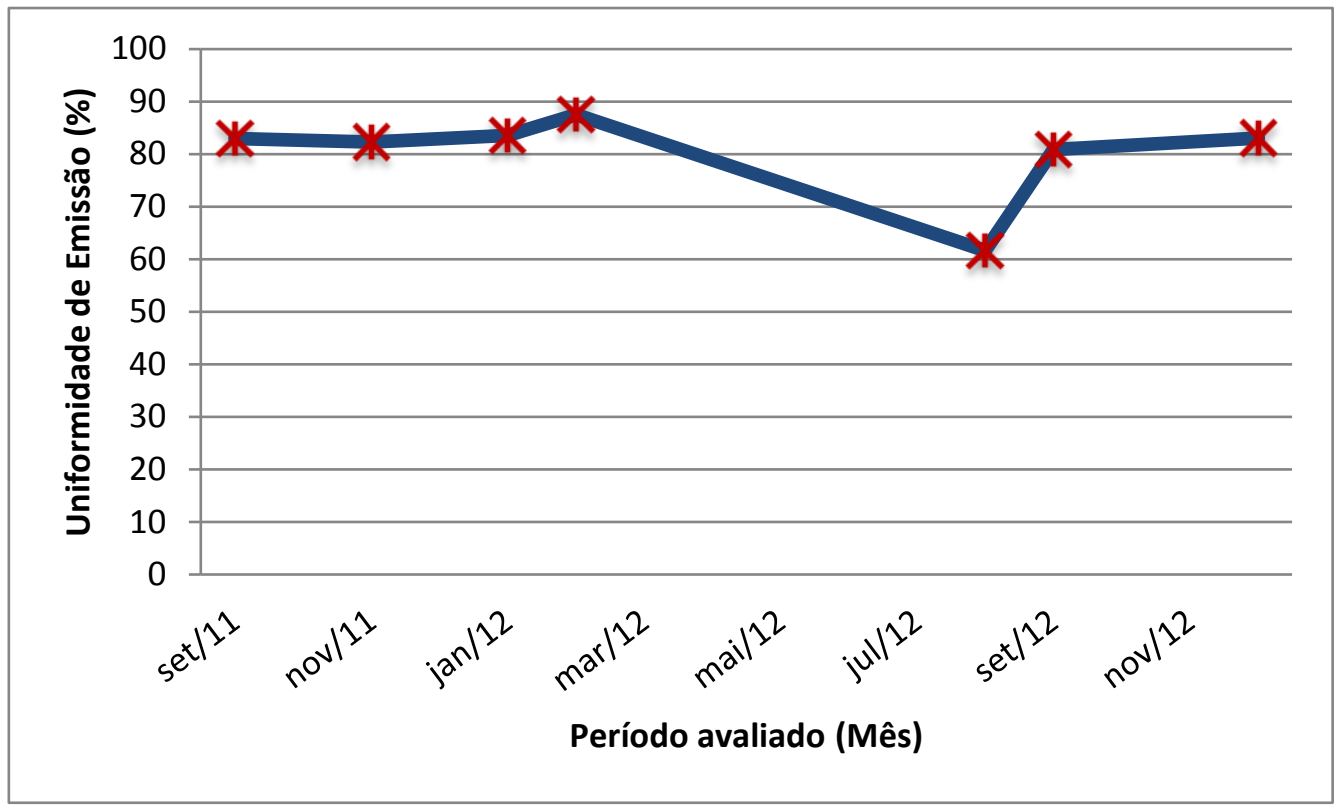

Figura 04. Evolução da qualidade da irrigação no DIBAU com o SAI

\section{CONCLUSÕES}

Concluímos que alguns fatores contribuem para uma baixa qualidade da irrigação como a falta de orientação por parte de um técnico especializado, longos períodos sem irrigação e, também, a falta de manutenção dos sistemas de irrigação. $\mathrm{O}$ irrigante tem

\section{AGRADECIMENTOS}

Agradecemos o apoio da Fundação de Amparo a Pesquisa do Estado de São Paulo - FAPESP e do Conselho Nacional de Desenvolvimento

\section{REFERÊNCIAS BIBLIOGRÁFICAS}

ALLEN RG, PEREIRA LS, RAES D, SMITH M., Crop evapotranspiration: guidelines for computing crop water requirements. Irrigation and Drainage. Paper 56. FAO, Rome, Italy, 1998

BRALTS, V.F. Field performance and evaluation. In: NAKAYAMA, F.S.; BUCKS, D.A. (Ed.) Trickle irrigation uma melhoria considerável quando é assessorado por um técnico e por tecnologia apropriada.

O SAI com o auxílio do s@i se mostraram eficientes e outras pesquisas esta linha deverão ser realizadas ao longo do projeto.

Científico e Tecnológico - CNPq, pelo financiamento de projeto de pesquisa coordenado pelo INCT em Engenharia da Irrigação (INCT-EI).

for crop production. Amsterdam: Elsevier, 1986. p.216-240.

CAR N. J., CHRISTEN E. W., HORNBUCKLE J. W., MOORE G. A. Using a mobile phone Short Messaging Service (SMS) for irrigation scheduling in Australia - Farmers' participation and utility evaluation, Computers and 


\section{EVOLUÇÃO DA QUALIDADE DA IRRIGAÇÃO APÓS AS ATUAÇÕES DO SERVIÇO DE ASSESSORAMENTO AO IRRIGANTE}

Electronics in Agriculture, 84, 132143,2012

FRIZZONE, J. A.; FREITAS, P. S. L.; REZENDE, R. ; FARIA, M. A. . Microirrigação: Gotejamento e Microaspersão. 1. ed. Maringá: Eduem - Editora da Universidade Estadual de Maringá, 2012. v. 1. 356p.

CÓRCOLES JI, DE JUAN JA, ORTEGA JF, TARJUELO JM, MORENO M. A. Management evaluation of Water Users Associations using benchmarking techniques. Agricultural Water Management 98:1-11, 2010

FRIZZONE, J.A. Planejamento da irrigação com uso de técnicas de otimização. Revista Brasileira de Agricultura Irrigada, Fortaleza, v. 1, n. 1, p. 24-49, dez. 2007.

GONZÁLEZ-DUGO, M.P., MATEOS, L., Spectral vegetation indices for benchmarking water productivity of irrigated cotton and sugarbeet crops. Agricultural Water Management 95, 48-58, 2008

KELLER, J.; KARMELI, D. Trickle irrigation design parameters. Transactions of the ASAE, St. Joseph, v.17, n.4, p.678-684, 1974.

LIMA, S. C. R. V.; FRIZZONE, J. A.; MATEOS, L.; FERNANDEZ. M. S. . Desempenho da Irrigação em um Projeto Hidroagrícola no Sul da Espanha: metodologia para análise da eficiência de uso da água. Revista Brasileira de Agricultura Irrigada, v.4, p. 59-77, 2010.

LIMA, S. C. R. V.; FRIZZONE, J. A.; SOUSA, A. E. C.; BELTRÃO JÚNIOR, J. A.; FERREIRA, R. P.; GARCIA, D. R. Aplicação da tecnologia da informação e a adoção pelo agricultor: a avaliação inicial do envio de mensagens pelo serviço de assessoramento ao irrigante. Revista Brasileira de Agricultura Irrigada, v.6, p. 314-328, 2012.

LIRA，J. V.; MOURÃO JÚNIOR, F.; SOUSA, A. E. C.; LIMA, S. C. R. V. ; FRIZZONE, J. A; VIANA, J. M. Auditoria de Desempenho de Sistemas de Irrigação I: A experiência inicial do Serviço de Assessoramento ao Irrigante - SAI, no Distrito de Irrigação do Baixo Acaraú. Revista Brasileira de Agricultura Irrigada, v.5, p. 265-271, 2011

LORITE I. J.; GARCÍA-VILA M.; CARMONA M. A.; SANTOS C.; SORIANO M. A. Assessment of the Irrigation Advisory Services' Recommendations and Farmers' Irrigation Management: A Case Study in Southern Spain Water Resources Management 26:2397-2419, 2012

MATEOS, L., Identifying a new paradigm for assessing irrigation system performance Irrigation Science, Amsterdam, v. 27, p. 25-34, 2008.

MONTORO A, LÓPEZ-FUSTER P, FERERES E. Improving on-farm water management through an irrigation scheduling service. Irrigation Science 29:311-319, 2011

ORTEGA J.F., DE JUAN J.A., TARJUELO J. M. Improving water management: The irrigation advisory service of Castilla-La Mancha (Spain). Agricultural Water Management 77:37-58, 2005

SANTOS NETO, A. M. DOS, BRAGA, A. C. C., SILVA, M. M. M. 3, LIMA, S. C. R. V., FRIZZONE, J. A., GOMES FILHO, R. R. Auditoria de 


\section{EVOLUÇÃO DA QUALIDADE DA IRRIGAÇÃO APÓS AS ATUAÇÕES DO} SERVIÇO DE ASSESSORAMENTO AO IRRIGANTE

Desempenho de Sistemas de Irrigação II: Avaliação e Correção da Uniformidade de Emissão de Água no Distrito de Irrigação do Baixo Acaraú, CE. Revista Brasileira de Agricultura Irrigada $v .5, n^{\circ} .4, p .272-279,2011$

WU, I.P. An assessment of hydraulic design of micro-irrigation systems. Agricultural Water Management, v.32, n.3, p.275-284, 1997.
WU, I.P.; BARRAGÁN, J.; BRALTS, V. Field performance and evaluation. In: LAMM, F.R.; AYARS, J.E.; KAKAYAMA, F.S. Microirrigation for crop production: design, operation, and management. Developments in Agricultural Engineering 13. Amsterdam: Elsevier, 2007. Cap.10, p.357-387. 